\title{
Non-Linear Stability of Conical Shell Structures Including a Functionally Graded Core
}

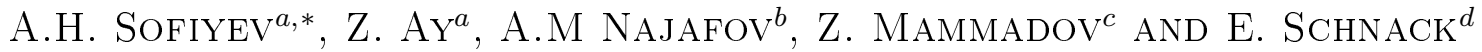 \\ ${ }^{a}$ Department of Civil Engineering, Suleyman Demirel University, Isparta, Turkey \\ ${ }^{b}$ Institute for Machine Elements and Lifting-and-Shifting Machines of Azerbaijan Technical University \\ Baku, Azerbaijan \\ ${ }^{c}$ Department of Civil Engineering, Harran University, Sanliurfa, Turkey \\ ${ }^{d}$ Institute of Solid Mechanics, Karlsruhe Institute of Technology, Karlsruhe, Germany
}

\begin{abstract}
In this study, the non-linear stability of three-layered conical shells with functionally graded core have not been investigated previously. The purpose of this paper is to study this problem. The large deflection theory with von Karman-Donnell-type of kinematic non-linearity is used to deduce the basic equations. The basic equations are solved analytically by using superposition and Galerkin methods. The influences of several parameters on the dimensionless non-linear critical axial loads are discussed.
\end{abstract}

DOI: $10.12693 /$ APhysPolA.125.192

PACS: $61.43 . \mathrm{Bn}, 62.20 . \mathrm{mq}$

\section{Introduction}

Layered composite structures are commonly used in many kinds of engineering structures. In conventional laminated composite structures, homogeneous elastic lamina are bonded together to obtain enhanced mechanical properties. However, the abrupt change in material properties across the interface between different materials can result in large inter-laminar stresses leading to delamination. One way to overcome these adverse effects is to use "functionally graded materials (FGMs)" in which material properties vary continuously. This may be achieved by gradually changing the volume fraction of the constituent materials, usually in the thickness direction only. This advantage eliminates interface problems of composite materials and thus the stress distribution becomes smooth. Used as interfacial zones and coatings, they can help to reduce mechanically and thermally induced stresses caused by the material property mismatch and to improve the bonding strength.

The concept of FGMs was first introduced by a group of Japanese scientists in 1984 [1]. Due to the increased relevance of the FGMs structural components in the design of aerospace structures, their non-linear (NL) behaviors have attracted the attention of many scientists $[2,3]$. From a technical point of view, to determine the NL critical load of truncated conical shells requires more complex mathematical operations and calculations. Thus the stability of FGM conical shells is quite limited in the geometrical NL formulation [4]. From the literature survey, one can see that the NL stability of layered conical shells with FG core have not been investigated previously. The purpose of this paper is to study this problem. The large deflection theory with von Karman-Donnell-type of

*corresponding author; e-mail: as of iyev@mmf . sdu.edu.tr kinematic non-linearity is used to deduce the basic equations. Basic equations are solved by using superposition and Galerkin methods.

\section{Formulation of the problem}

Figure 1 shows a three-layered truncated conical shell with the FG core and given the nomenclature. Here $h$ is the total thickness of the three-layered truncated conical shell, $h_{\mathrm{FG}}=2 a$ is the thickness of a FG core, $L$ and $\gamma$ are the length and semi-vertex angle truncated cone, respectively, and $u, v, w$ are displacements. The vertical ordinates of the top, the two interfaces, and the bottom are denoted by $h_{1}=-h / 2, h_{2}=-a, h_{3}=a, h_{4}=h / 2$, respectively. The compositional gradation of a FG core is defined by the volume fraction of the ceramic phase and the inverse quadratic function will be considered, $V(\bar{\zeta})=1-(0.5-\bar{\zeta})^{2}$, here $\bar{\zeta}=\zeta / h_{\mathrm{Fg}}$ and $\zeta$ is the thickness coordinate. For such cases, the variation of the Young modulus and Poisson ratio in the three-layer system are given as

$$
\begin{aligned}
& {[E(\bar{\zeta}), \nu(\bar{\zeta})]=} \\
& \left\{\begin{array}{l}
E_{\mathrm{m}}, \nu_{\mathrm{m}}, \quad-h_{1} / h_{\mathrm{FG}} \leq \bar{\zeta} \leq-h_{2} / h_{\mathrm{FG}}, \\
E_{\mathrm{fg}}=\left(E_{\mathrm{c}}-E_{\mathrm{m}}\right)\left[1-(0.5-\bar{\zeta})^{2}\right]+E_{\mathrm{m}} ; \\
\nu_{\mathrm{fg}}=\left(\nu_{\mathrm{c}}-\nu_{\mathrm{m}}\right)\left[1-(0.5-\bar{\zeta})^{2}\right]+\nu_{\mathrm{m}}, \\
\quad-h_{2} / h_{\mathrm{FG}} \leq \bar{\zeta} \leq h_{3} / h_{\mathrm{FG}}, \\
E_{\mathrm{c}}, \nu_{\mathrm{c}}, \quad h_{3} / h_{\mathrm{FG}} \leq \bar{\zeta} \leq h_{4} / h_{\mathrm{FG}} .
\end{array}\right.
\end{aligned}
$$

The non-linear basic equations of three-layered truncated conical shells with a FG core can be written in the form as

$$
\begin{gathered}
c_{2} \mathrm{e}^{2 x}\left(\frac{\partial^{4} \Phi_{1}}{\partial x^{4}}-4 \frac{\partial^{3} \Phi_{1}}{\partial x^{3}}+4 \frac{\partial^{2} \Phi_{1}}{\partial x^{2}}+\frac{\partial^{4} \Phi_{1}}{\partial \varphi^{4}}+2 \frac{\partial^{2} \Phi_{1}}{\partial \varphi^{2}}\right) \\
+\left(\frac{\partial^{2} \Phi_{1}}{\partial x^{2}}+3 \frac{\partial \Phi_{1}}{\partial x}+2 \Phi_{1}\right) S_{1} \mathrm{e}^{3 x} \cot \gamma
\end{gathered}
$$




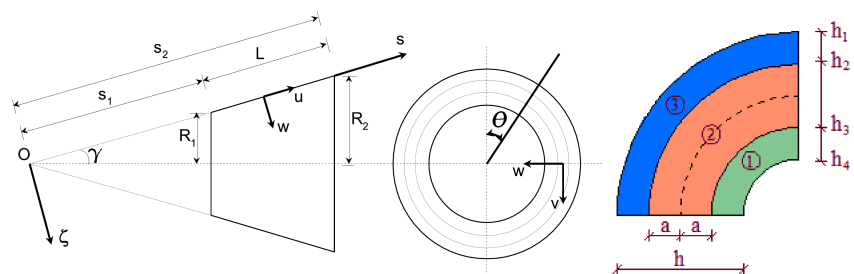

Fig. 1. The cross-section of three-layered conical shell with a FG core and nomenclature.

$$
\begin{aligned}
& +2\left(c_{1}-c_{5}\right) \mathrm{e}^{2 x}\left(\frac{\partial^{4} \Phi_{1}}{\partial x^{2} \partial \varphi^{2}}+2 \frac{\partial^{3} \Phi_{1}}{\partial x \partial \varphi^{2}}+\frac{\partial^{2} \Phi_{1}}{\partial \varphi^{2}}\right) \\
& -c_{3}\left(\frac{\partial^{4} w}{\partial \varphi^{4}}+2 \frac{\partial^{2} w}{\partial \varphi^{2}}+\frac{\partial^{4} w}{\partial x^{4}}-4 \frac{\partial^{3} w}{\partial x^{3}}+4 \frac{\partial^{2} w}{\partial x^{2}}\right) \\
& -2\left(c_{4}+c_{6}\right)\left(\frac{\partial^{4} w}{\partial x^{2} \partial \varphi^{2}}-2 \frac{\partial^{3} w}{\partial x \partial \varphi^{2}}+\frac{\partial^{2} w}{\partial \varphi^{2}}\right) \\
& +\mathrm{e}^{2 x}\left(\frac{\partial^{2} \Phi_{1}}{\partial \varphi^{2}}+\frac{\partial \Phi_{1}}{\partial x}+2 \Phi_{1}\right)\left(\frac{\partial^{2} w}{\partial x^{2}}-\frac{\partial w}{\partial x}\right) \\
& +\mathrm{e}^{2 x}\left(\frac{\partial^{2} \Phi_{1}}{\partial x^{2}}+3 \frac{\partial \Phi_{1}}{\partial x}+2 \Phi_{1}\right)\left(\frac{\partial^{2} w}{\partial \varphi^{2}}+\frac{\partial w}{\partial x}\right) \\
& -2 \mathrm{e}^{2 x}\left(\frac{\partial^{2} \Phi_{1}}{\partial x \partial \varphi}+\frac{\partial \Phi_{1}}{\partial \varphi}\right)\left(\frac{\partial^{2} w}{\partial x \partial \varphi}-\frac{\partial w}{\partial \varphi}\right)=0, \\
& b_{1} \mathrm{e}^{2 x} \frac{\partial^{4} \Phi_{1}}{\partial \varphi^{4}}+2\left(b_{5}+b_{2}\right) \mathrm{e}^{2 x} \frac{\partial^{4} \Phi_{1}}{\partial x^{2} \partial \varphi^{2}} \\
& -4\left(b_{5}+b_{2}\right) \mathrm{e}^{2 x} \frac{\partial^{3} \Phi_{1}}{\partial x \partial \varphi^{2}}+2\left(b_{5}+b_{2}+b_{1}\right) \mathrm{e}^{2 x} \frac{\partial^{2} \Phi_{1}}{\partial \varphi^{2}} \\
& +b_{1} \mathrm{e}^{2 x} \frac{\partial^{4} \Phi_{1}}{\partial x^{4}}-4 b_{1} \mathrm{e}^{2 x} \frac{\partial^{3} \Phi_{1}}{\partial x^{3}}+4 b_{1} \mathrm{e}^{2 x} \frac{\partial^{2} \Phi_{1}}{\partial x^{2}} \\
& -b_{4} \frac{\partial^{4} w}{\partial \varphi^{4}}-2\left(b_{3}-b_{6}\right) \frac{\partial^{4} w}{\partial x^{2} \partial \varphi^{2}}-b_{4} \frac{\partial^{4} w}{\partial x^{4}} \\
& -4\left(b_{6}-b_{3}\right) \frac{\partial^{3} w}{\partial x \partial \varphi^{2}}-2\left(b_{3}-b_{6}+b_{4}\right) \frac{\partial^{2} w}{\partial \varphi^{2}} \\
& -4 b_{4} \frac{\partial^{3} w}{\partial x^{3}}-\left(4 b_{4}-S_{1} \mathrm{e}^{x} \cot \gamma\right) \frac{\partial^{2} w}{\partial x^{2}} \\
& -S_{1} \mathrm{e}^{x} \cot \gamma \frac{\partial w}{\partial x}-\left(\frac{\partial w}{\partial \varphi}\right)^{2}+2 \frac{\partial w}{\partial \varphi} \frac{\partial^{2} w}{\partial x \partial \varphi} \\
& -\left(\frac{\partial w}{\partial x}-\frac{\partial^{2} w}{\partial x^{2}}\right) \frac{\partial^{2} w}{\partial \varphi^{2}}-\left(\frac{\partial^{2} w}{\partial x \partial \varphi}\right)^{2} \\
& -\left(\frac{\partial w}{\partial x}-\frac{\partial^{2} w}{\partial x^{2}}\right) \frac{\partial w}{\partial x}=0,
\end{aligned}
$$

where $c_{i}, b_{i}, i=1,2, \ldots, 6$ are parameters, which included the following definitions:

$$
A_{1 k_{1}}=\frac{E_{\mathrm{m}}}{1-\nu_{\mathrm{m}}^{2}} \int_{-h_{1}}^{-h_{2}} \zeta^{k_{1}} \mathrm{~d} \zeta+\int_{-h_{2}}^{h_{3}} \zeta^{k_{1}} \frac{E_{\mathrm{fg}}}{1-\nu_{\mathrm{fg}}^{2}} \mathrm{~d} \zeta
$$

$$
\begin{gathered}
+\frac{E_{\mathrm{c}}}{1-\nu_{\mathrm{c}}^{2}} \int_{h_{3}}^{h_{4}} \zeta^{k_{1}} \mathrm{~d} \zeta, \\
A_{2 k_{1}}=\frac{\nu_{\mathrm{m}} E_{\mathrm{m}}}{1-\nu_{\mathrm{m}}^{2}} \int_{-h_{1}}^{-h_{2}} \zeta^{k_{1}} \mathrm{~d} \zeta+\int_{-h_{2}}^{h_{3}} \zeta^{k_{1}} \frac{\nu_{\mathrm{fg}} E_{\mathrm{fg}}}{1-\nu_{\mathrm{fg}}^{2}} \mathrm{~d} \zeta \\
+\frac{\nu_{\mathrm{c}} E_{\mathrm{c}}}{1-\nu_{\mathrm{c}}^{2}} \int_{h_{3}}^{h_{4}} \zeta^{k_{1}} \mathrm{~d} \zeta, \\
A_{6 k_{1}}=\frac{E_{\mathrm{m}}}{1+\nu_{\mathrm{m}}} \int_{-h_{1}}^{-h_{2}} \zeta^{k_{1}} \mathrm{~d} \zeta+\int_{-h_{2}}^{h_{3}} \zeta^{k_{1}} \frac{E_{\mathrm{fg}}}{1+\nu_{\mathrm{fg}}} \mathrm{d} \zeta \\
\quad+\frac{E_{\mathrm{c}}}{1+\nu_{\mathrm{c}}} \int_{h_{3}}^{h_{4}} \zeta^{k_{1}} \mathrm{~d} \zeta, \quad k_{1}=0,1,2 .
\end{gathered}
$$

\section{Solution of basic equations}

Due to the three-layered truncated conical shell with a FG core is satisfied simply-supported boundary conditions, the solution of Eq. (3) is sought in the following form:

$$
w=f_{1} \mathrm{e}^{x} \sin \left(m_{1} x\right) \sin \left(m_{2} \varphi\right)+f_{2} \sin ^{2}\left(m_{1} x\right),
$$

where $f_{1}$ and $f_{2}$ are unknown amplitudes of the displacement $w$. Here, the first term corresponds to that used in the buckling theory of infinitesimal deflections. The second term reflects the preferred inward bulging of the conical shell when the displacements become large and the following definitions apply: $m_{1}=m \pi / x_{0}, m_{2}=n / \sin \gamma$, $x_{0}=\ln \left(S_{2} / S_{1}\right)$, where $m$ is the number of half-waves along a generatrix and $n$ is the number of full-waves along a parallel circle.

$$
\begin{aligned}
\Phi_{1} & =K_{1} \mathrm{e}^{-x} f_{1} \sin \left(m_{1} x\right) \sin \left(m_{2} \varphi\right) \\
& +K_{2} \mathrm{e}^{-x} f_{1} \cos \left(m_{1} x\right) \sin \left(m_{2} \varphi\right) \\
& +f_{2} \mathrm{e}^{-x}\left[K_{3} \cos \left(2 m_{1} x\right)+K_{4} \sin \left(2 m_{1} x\right)\right] \\
& \times\left[K_{51} f_{2}^{2}+K_{52} f_{1}^{2}+K_{53} f_{2}\right] \cos \left(2 m_{1} x\right) \\
& +\left(K_{61} f_{2}^{2}+K_{62} f_{1}^{2}+K_{63} f_{2}\right) \sin \left(2 m_{1} x\right) \\
& +f_{1}^{2} \cos \left(2 m_{2} \varphi\right)\left[K_{7} \cos \left(2 m_{1} x\right)+K_{8} \sin \left(2 m_{1} x\right)\right] \\
& +\left(K_{91} f_{2}+K_{92}\right) f_{1} \cos \left(m_{1} x\right) \sin \left(m_{2} \varphi\right) \\
& +f_{1}\left[K_{101} f_{2}(t)+K_{102}\right] \sin \left(m_{1} x\right) \sin \left(m_{2} \varphi\right) \\
& +f_{1} f_{2} \sin \left(m_{2} \varphi\right)\left[K_{11} \cos \left(3 m_{1} x\right)+K_{12} \sin \left(3 m_{1} x\right)\right] \\
& +f_{2}^{2}\left[K_{13} \cos \left(4 m_{1} x\right)+K_{14} \sin \left(4 m_{1} x\right)\right] \\
& +K_{15} f_{1}^{2} \cos \left(2 m_{2} \varphi\right)+K_{16} f_{2} \mathrm{e}^{-x} \\
& -\left[1+\mathrm{e}^{2 x}+\cos \left(m_{2} \varphi\right)\right] T_{0} S_{1}^{2} / 2
\end{aligned}
$$

where $T_{0}$ is axial load, $K_{i}(i=1,2, \ldots, 102)$ are parameters depending on FG core properties and shell characteristics [4].

Taking into account (5) and (6) in Eq. (2) and minimizing resulting equation depending on $\bar{f}_{1}^{2}$, for the dimen- 
sionless NL critical axial load of three-layered truncated conical shell with a FG core, the following expression is obtained:

$$
T_{1 \mathrm{cr}}^{\mathrm{NL}}=\frac{4 \bar{B}_{13} \bar{B}_{11} \lambda^{2}+\left(\bar{B}_{14}+\bar{B}_{12} \lambda\right)^{2}}{4 \bar{B}_{13} \lambda^{2}},
$$

where the following definitions apply:

$$
T_{1 \mathrm{cr}}^{\mathrm{NL}}=T_{0 \mathrm{cr}}^{\mathrm{NL}} / E_{\mathrm{c}} h, \bar{B}_{1 j}=B_{1 j} / E_{\mathrm{c}} h, j=1,2, \ldots, 6 .
$$

In determination of the lower limit of the nonlinear stability, Eq. (7) minimized with respect to wave numbers $(m, n)$.

As the non-linear terms are neglected in Eq. (7), the following expression is obtained for the dimensionless linear critical axial load of the truncated conical shell with a FG core:

$$
T_{1 \mathrm{cr}}^{\operatorname{lin}}=-B_{11} / E_{\mathrm{c}} h .
$$

\section{Numerical computations and results}

In this subsection, the numerical computations, for dimensionless L and NL critical axial loads of the pure metal, pure ceramic, pure FG truncated conical shells and three-layered truncated conical shells with a FG core have been carried out using expressions (7) and (9), respectively. A computer program called Maple 14 has been used to compute numerical values from the formulae obtained. An FG core prepared with the mixture of the silicon nitride and titanium alloys and referred to as $\mathrm{Si}_{3} \mathrm{~N}_{4} / \mathrm{Ti}-6 \mathrm{Al}-4 \mathrm{~V}$ or FG. The effective material properties for selected FG core are given in [4]. The three- -layered conical shell formed as $\mathrm{Si}_{3} \mathrm{~N}_{4} / \mathrm{FG} / \mathrm{Ti}-6 \mathrm{Al}-4 \mathrm{~V}$ or $\mathrm{S} / \mathrm{FG} / \mathrm{T}$. The materials are assumed to be perfectly elastic throughout the deformation. The variations of the values of $T_{1 \mathrm{cr}}^{\mathrm{NL}}$ and $T_{1 \mathrm{cr}}^{\mathrm{L}}$ for the single-layer $\mathrm{Si}_{3} \mathrm{~N}_{4}$ and Ti-6Al-4V, the pure FG and the three-layered S/FG/T truncated conical shells, versus the ratio of total thickness of the shell to thickness of a FG core, $h / h_{\mathrm{FG}}$, are shown in Table. The three-layered truncated conical shell transformed to the single-layer pure FG truncated conical shell, as $h / h_{\mathrm{FG}}=1$. The $\mathrm{FG}$ core is taken to be inverse-quadratic compositional profile and the shell characteristics are: $R_{1} / h=150, \gamma=45^{\circ}, L / R_{1}=2$. It is seen that the values of $T_{1 \mathrm{cr}}^{\mathrm{NL}}$ and $T_{1 \mathrm{cr}}^{\mathrm{L}}$ for three-layered $\mathrm{S} / \mathrm{FG} / \mathrm{T}$ truncated conical shell decrease, as the ratio $h / h_{\mathrm{FG}}$ increases from 2 to 5 . It is observed that the ratio, $T_{1 \mathrm{cr}}^{\mathrm{NL}} / T_{1 \mathrm{cr}}^{\mathrm{L}}$, for the three-layered $\mathrm{S} / \mathrm{FG} / \mathrm{T}$ truncated conical shell is nearly 0.48 , as $h / h_{\mathrm{FG}}$ increases from 2 to 5 . As the three-layered S/FG/T truncated conical shell with FG-inverse quadratic core are compared with the single-layer pure FG-inverse quadratic truncated conical shell, the influence of the inverse quadratic compositional profile on $T_{1 \mathrm{cr}}^{\mathrm{NL}}$ and $T_{1 \mathrm{cr}}^{\mathrm{L}}$ increases, as $h / h_{\mathrm{FG}}$ increases from 2 to 5 . In addition, the maximum effect on the values of dimensionless L and NL critical axial loads for $\mathrm{S} / \mathrm{FG} / \mathrm{T}$ truncated conical shell are $15.75 \%$ and $16.36 \%$, respectively. Consequently, the effect of the ratio $h / h_{\mathrm{FG}}$ is more pronounced for $T_{1 \mathrm{cr}}^{\mathrm{NL}}$ than $T_{1 \mathrm{cr}}^{\mathrm{L}}$. As $\mathrm{S} / \mathrm{FG} / \mathrm{T}$ is compared with the pure ceramic and pure metal truncated conical shells, respectively, the influence of the inverse quadratic compositional profile on the dimensionless critical loads decreases and increases, respectively, as $h / h_{\mathrm{FG}}$ increases.

\section{TABLE}

Variations of the dimensionless L and NL critical axial loads, their ratios and corresponding wave numbers for the single-layer pure ceramic, pure metal, pure FG and three-layered S/FG/T truncated conical shells versus $h / h_{\mathrm{FG}}$.

\begin{tabular}{c|c|c|c|c|c|c}
\hline \hline \multirow{2}{*}{$h / h_{\mathrm{FG}}$} & $T_{1 \mathrm{cr}}^{\mathrm{L}} \times 10^{3}(m, n)$ & $T_{1 \mathrm{cr}}^{\mathrm{NL}} \times 10^{3}(m, n)$ & $T_{1 \mathrm{cr}}^{\mathrm{L}} \times 10^{3}(m, n)$ & $T_{1 \mathrm{cr}}^{\mathrm{NL}} \times 10^{3}(m, n)$ & $T_{1 \mathrm{cr}}^{\mathrm{L}} \times 10^{3}(m, n)$ & $T_{1 \mathrm{cr}}^{\mathrm{NL}} \times 10^{3}(m, n)$ \\
\cline { 2 - 6 } & \multicolumn{2}{|c|}{$\mathrm{Si}_{3} \mathrm{~N}_{4}$} & \multicolumn{2}{|c|}{$\mathrm{S} / \mathrm{FG} / \mathrm{T}$} & \multicolumn{2}{|c}{$\mathrm{Ti}-6 \mathrm{Al}-4 \mathrm{~V}$} \\
\hline 1 & & & $1.105(10,6)$ & $0.538(5,22)$ & & \\
2 & \multirow{3}{*}{$1.508(10,3)$} & $0.715(2,10)$ & $0.978(10,6)$ & $0.476(8,21)$ & $0.503(10,2)$ & $0.233(9,26)$ \\
3 & & & $0.950(10,7)$ & $0.456(8,21)$ & & \\
5 & & & $0.931(10,7)$ & $0.450(8,21)$ & &
\end{tabular}

\section{Conclusions}

The NL stability analysis is presented for axially compressed truncated conical shells with the FG core. The mechanical properties of FG core are assumed to be graded in the thickness direction according to an inverse quadratic distribution. The governing equations of the three-layered truncated conical shell with a FG core are derived using the large deformation theory with von Karman-Donnell-type of kinematic non-linearity and are solved by using superposition and Galerkin methods. The numerical results reveal that the variations of the shell thickness-to-FG core thickness ratio and the com- positional profile have significant effects on L and NL critical axial loads of three-layered truncated conical shells with a FG core. The results are verified by comparing the obtained values with those in the existing literature.

\section{References}

[1] M. Koizumi, Compos. 28, 1 (1997).

[2] H.W. Huang, Q. Han, Int. J. Mech. Sci. 51, 500 (2009).

[3] K.M. Liew, K.M.X. Zhao, Y.Y. Lee, Compos. B Eng. 43, 1621 (2012).

[4] A.H. Sofiyev, Int. J. Nonlin. Mech. 46, 711 (2011). 